\title{
COMPRENDER LA URBANIDAD. OPORTUNIDADES Y DESAFÍO DE LA ANTROPOLOGÍA URBANA EN EL MARCO DE LA POLIITICA NACIONAL DE DESARROLLO URBANO.
}

\author{
Understanding urbanity. Opportunities and challenge of Urban Anthropology in the \\ framework of the National Urban Development Policy.
}

\section{CARLOS LANGE VALDÉS*}

Fecha de recepción: 5 de marzo de 2015 - Fecha de aprobación: 11 de mayo 2015

\section{Resumen}

Durante los últimos años se ha desarrollado en Chile el proceso de discusión y formulación de la Política Nacional de Desarrollo Urbano, la cual se encuentra actualmente en etapa de implementación. Dicho proceso ha contemplado mínimamente los aportes provenientes desde las ciencias sociales y particularmente desde la antropología urbana, lo cual refleja la escasa incidencia de la disciplina en los procesos de desarrollo urbano. Sin embargo, más que una lectura pesimista de aquello, en el presente artículo se considera que este proceso ofrece oportunidades y un desafío para la identificación de nuevos temas que permitan consolidar y expandir nuestro campo de conocimiento disciplinar en torno al concepto de urbanidad.

Palabras clave: urbanidad, desarrollo urbano, cultura urbanística.

\section{Abstract}

In recent years, in Chile has been developed a process of discussion and formulation of the National Urban Development Policy, which is currently being implemented. This process has contemplated minimun contributions from the social sciences and particularly from urban anthropology, reflecting the low incidence of discipline in urban development processes. However, rather than a pessimistic view, in this article we consider that this process provides opportunity and challenge, for the identification of new issues that allow consolidate and expand our field of disciplinary knowledge on the concept of urbanity.

Keywords: urbanity, urban development, town-planning culture.

\footnotetext{
* Licenciado en Antropología, Universidad de Chile. Mg Desarrollo Urbano, PUC. Doctor en Ciencias Sociales, Universidad de Deusto (España). Académico INVI-FAU. Correo electrónico: clange@uchilefau.cl
} 


\section{Introducción}

En un texto publicado hace un poco más de diez años (Imilan \& Lange, 2003) se planteaba que el campo de la antropología urbana en Chile poseía por esos años un carácter incipiente y difuso, el cual transitaba aún en torno a la clásica distinción entre antropología en la ciudad y antropología de la ciudad. Dicho diagnóstico aparecía sustentado en torno a dos consideraciones claves: por una parte, la marcada tendencia de la práctica disciplinar a entramparse en torno a la búsqueda y el análisis de grupos considerados como unidades sociales con una cierta homogeneidad interna, vinculados a un territorio y con un fuerte componente comunitario, lo cual impedía el estudio de los cruces e intersecciones culturales que constituyen la condición propia de lo urbano; por otro, la existencia de un objeto de conocimiento -lo urbano- escasamente comprendido y abordado en el marco de ciudades constituidas más como agregados de formas, funciones y personas, que como artefactos cuyas configuraciones formales, funcionales y culturales responden a una lógica interna claramente identificable y con una dinámica de reproducción particular.

En comparación a este diagnóstico, no cabe duda que durante los últimos diez años la antropología urbana en Chile ha experimentado un creciente proceso de maduración, convirtiéndose en un campo de especialización cada vez más afianzado. Por una parte, se ha ampliado la oferta académica tanto a nivel de pregrado como de posgrado, abriendo nuevos espacios para el desarrollo de la antropología urbana como línea de especialización. Asimismo, se han ampliado y diversificado los dispositivos que permiten producir y difundir su quehacer, abordándose no solamente a través de cáte- dras regulares sino también a través de proyectos de investigación, grupos y redes de trabajo, presentaciones en congresos, coloquios, seminarios, difusión a través de medios impresos y digitales, entre otros. Lo anterior ha permitido ampliar sus perspectivas hacia nuevos temas de investigación que acercan a la antropología hacia otras disciplinas afines al campo de lo urbano, como por ejemplo la sociología, la geografía, la arquitectura, entre otras. Uno de estos temas es la conformación de la urbanidad, entendida como el campo de relaciones sociales característico de los espacios urbanizados.

Tal como lo ha planteado Giglia la urbanidad ha sido comúnmente entendida como "el código de las buenas maneras y vehículo de la distinción social" (2001:806). Esta noción permite dar cuenta de las formas de comportamiento apropiadas para estar en el ámbito público, permitiendo a sujetos social y culturalmente distintos poder convivir en la ciudad atendiéndose mutuamente y relacionándose entre sí. En tal sentido, e interpretando a la autora, la urbanidad integra relacionalmente aquellos componentes de la vida social urbana sin desconocer su evidente connotación política ${ }^{1}$.

Sin embargo, esta connotación política no se sustenta en acuerdos racional e institucionalmente establecidos, sino que surge de forma emergente. En esta perspectiva, y siguiendo a Joseph (2002), la noción de urbanidad puede ser considerado un interesante corolario de la dinámica creadora y distintiva de la vida social urbana $^{2}$. Y si bien el nivel de influencia que las ciencias sociales en general, y la antropología urbana en particular han tenido sobre los procesos de desarrollo urbano, en nuestro país ha sido tradicionalmente escaso, comprender la urbanidad puede abrirse como un importante ámbito para el desarrollo de nuestra disciplina 
así como también una interesante contribución de ésta al campo de conocimiento de lo urbano.

En este sentido, y tal como ha planteado Capel (2003), para abordar los procesos de desarrollo urbano resulta fundamental considerar la necesaria articulación entre urbs, polis y civitas. Para el autor, la urbs alude al espacio construido, es decir al conjunto de infraestructuras, equipamientos y servicios que sustentan y distinguen física y materialmente la forma espacial y arquitectónica de la ciudad; por su parte, la polis dice relación con la conformación de formas de organización político-administrativas que regulan y ordenan su funcionamiento; a su vez, la civitas alude a las distintas expresiones de la vida social desarrollada por los habitantes de éstas. La articulación entre estas nociones permite comprender que el desarrollo de los centros urbanos en la actualidad no pasa solamente por la provisión de infraestructuras, equipamientos y servicios tecnológicamente avanzados, económicamente productivos, financieramente rentables y estéticamente atractivos, sino que es necesario tender también hacia políticas de desarrollo urbano consensuadas y compartidas entre los distintos agentes sociales de manera que éstas sean sustentables a lo largo del tiempo. Asimismo, permite pensar que la implementación de políticas de desarrollo urbano no solamente se sustenta a partir de la formulación de normas y procedimientos que regulen su funcionamiento sino que ellas deben ser reconocidas y apropiadas por estos, así como también por los habitantes que representan. En esta línea, resulta relevante poner como ejemplo para el análisis el proceso de discusión, promulgación e implementación de la Política Nacional de Desarrollo Urbano (PNDU) en nuestro país, en la medida que éste constituye un claro ejemplo de la forma como se están pensando nuestras ciu- dades desde la perspectiva de aquellos agentes sociales urbanos que tradicionalmente han detentado mayores niveles de influencia en el campo de lo urbano.

Tal como se señala explícitamente en sus Objetivos y Principios, la PNDU debe "dar cabida a los distintos modos de vida", promoviendo el "concurso de la ciudadanía" en la toma de decisiones y fomentando el "sentido de pertenencia y la consecuente responsabilidad de la comunidad" con su entorno (MINVU, 2014). No obstante esta declaración de intenciones, desde nuestra perspectiva carece justamente de una cabal consideración a la importancia que la urbanidad posee como componente fundamental para el desarrollo urbano. En tal sentido, comprender la urbanidad como un campo amplio, diverso y dinámico de relaciones sociales que se establece y reproduce entre quienes habitan y comparten espacios urbanos a través de sus prácticas sociales resulta fundamental para avanzar en dicho camino, articulando las dimensiones de la urbs, polis y civitas señaladas por Capel y empoderando a los habitantes como agentes protagonistas de los procesos de desarrollo urbano.

\section{Breve descripción de los objetivos y ámbitos de la PNDU.}

Un primer antecedente relevante para comprender la importancia del proceso de formulación y actual implementación de la Política Nacional de Desarrollo Urbano es la premisa en torno a la ausencia de una política urbana precedente. En tal sentido, en abril de 2012 y bajo la convocatoria del gobierno de Sebastián Piñera, se conformó una Comisión Asesora Presidencial con el objetivo de formular una propuesta de política urbana que fuera capaz 
de guiar el desarrollo futuro de las ciudades y centros poblados del país. Tal como se declara en su presentación, su objetivo era desarrollar "un mandato compuesto por principios, objetivos y líneas de acción capaces de congregar voluntades, para luego concretarse en leyes, reglamentos y programas públicos orientados a lograr una mejor calidad de vida" (Op.cit.: 7).

Dicha Comisión Asesora Presidencial estuvo conformada por 28 personas provenientes de distintas áreas de la sociedad y del conocimiento, como representantes gremiales, decanos de universidades, parlamentarios, ex ministros, representantes de organizaciones ciudadanas, ministros en ejercicio, y profesionales expertos en diferentes materias atingentes al desarrollo urbano y territorial, aunque mayoritariamente provenientes de disciplinas como la arquitectura, ingeniería y el derecho. Su labor se desarrolló a través de distintas instancias de trabajo, considerando reuniones plenarias y de distintas subcomisiones, talleres ciudadanos regionales, encuentros intersectoriales ministeriales e instancias de participación virtual mediante plataformas digitales. Como resultado de dicha labor, el 4 de Marzo de 2014 fue publicada en el Diario Oficial la nueva Política Nacional de Desarrollo Urbano.

Asimismo, y como parte de su mandato, la PNDU establecía la necesidad de crear un Consejo de carácter consultivo y asesor, el cual de forma permanente e indefinida en el tiempo asumiera la tarea de impulsar su implementación a través de propuestas de reformas en los distintos ámbitos abordados por ésta, y de verificar el avance y el cumplimiento de los objetivos y lineamientos establecidos en ella. Este Consejo Nacional de Desarrollo Urbano (CNDU), constituido bajo el segundo gobierno de Michelle Bachelet, fue promulgado el 29 de abril de 2014 siendo conformado por 26 personas procedentes de distintos ámbitos vinculados al desarrollo urbano, como ministros, subsecretarios, senadores, diputados, alcaldes, dirigentes gremiales, académicos, representantes de la sociedad civil, entre otros. Éste fue organizado a partir de una Secretaría Ejecutiva y 6 Grupos de Trabajo en torno a materias como Política de Suelo e Integración Social, Nueva Institucionalidad del Desarrollo Urbano, Planificación Urbana Integrada, Identidad y Patrimonio Urbano, Equilibrio Ambiental Urbano y Desarrollo Económico Urbano.

La constitución y funcionamiento del CNDU también constituye un antecedente relevante para comprender la importancia del proceso en curso en la medida que su trabajo definirá orientaciones que, se espera, tengan incidencia en el desarrollo urbano y territorial a través de la propuesta de reformas y perfeccionamientos institucionales, legales y técnicas. Algunas de estas orientaciones se expresan en los avances ya alcanzados por los Grupos de Trabajo y expresadas en sus respectivos documentos de trabajo, en el desarrollo de estudios actualmente en curso respecto de estas temáticas y en instancias internacionales como la propuesta oficial chilena para la Tercera Conferencia de las Naciones Unidas sobre Asentamientos Humanos y Desarrollo Sustentable Hábitat III a desarrollarse en 2016.

Un primer paso en este sentido, es conocer los objetivos y lineamientos contenidos en la formulación de la PNDU. En una de sus definiciones más relevantes, establece la calidad de vida como el objetivo principal de su formulación y la sustentabilidad como el enfoque apropiado para conseguirla, promoviendo los principios del "bien común"3. Asimismo, se plantean cinco ámbitos temáticos que ordenan los obje- 
tivos específicos de esta política así como las líneas de acción asociados a ellos. Si bien estos poseen el nivel de generalidad característico de estas instancias, las cuales quedan supeditadas a sus procesos de implementación, en su formulación existe una escasa consideración a la relevancia que la urbanidad puede tener para la consecución de cada uno de ellos.

El primer ámbito temático abordado por la PNDU es el de la Integración Social, cuyo principal objetivo es "velar porque nuestras ciudades sean lugares inclusivos, donde las personas estén y se sientan protegidas e incorporadas a los beneficios urbanos" (Ídem: 31 ). Su relevancia radica en el explícito reconocimiento realizado en torno a los altos grados de desigualdad urbana y segregación social que presentan las ciudades chilenas, lo cual redunda en un conjunto de problemáticas sociales acuciantes como son la concentración de pobreza, la inseguridad, la falta de acceso a servicios públicos y escasa conectividad, entre otros.

En tal sentido, se propone revertir las actuales condiciones que propician esta problemática ya sea a través del establecimiento de estándares mínimos de habitabilidad, la promoción de programas públicos de integración social urbana, el establecimiento y consideración de criterios cuantitativos y cualitativos para la reducción del déficit habitacional, el establecimiento de una política de suelo regule normativamente su disponibilidad para vivienda social, el fomento de programas que fortalezcan la vida en comunidad y la conciencia ciudadana, entre otros, todos los cuales promuevan el acceso a los beneficios urbanos como los espacios públicos, educación, salud, trabajo, seguridad, interacción social, movilidad y transporte, cultura, deporte y esparcimiento, que permitan reducir los altos niveles de pobreza, desigualdad y exclusión social presentes en nuestro país.
El segundo ámbito temático está orientado a promover el Desarrollo Económico planteándose como objetivo principal la necesidad de "reforzar a las ciudades como agentes de desarrollo económico, fuentes de innovación, emprendimiento y creación de empleo" (Ídem: 39).

Destacan en este sentido, su orientación a generar condiciones que promuevan la innovación y creación de empleo como usos de suelos mixtos, mejores condiciones de infraestructura y equipamiento; la integración entre planificación urbana y programas de inversión, incorporando criterios de rentabilidad social, estableciendo incentivos a la inversión, planes de obras de interés público, entre otras; una mayor consideración a los efectos sociales en los proyectos públicos y privados corrigiendo imperfecciones de los mercados de suelos; el mejoramiento de las condiciones de competitividad de las ciudades; el establecimiento de reglas que otorguen certeza al desarrollo de proyectos; el mejoramiento de la calidad de los Instrumentos de Planificación Territorial, incorporando variables de desempeño económico y sistemas de evaluación de estas; una mayor eficiencia de las inversiones de infraestructura pública, considerando por ejemplo criterios de integración regional; una racionalización de los costos de transporte de personas y de bienes, regulando por ejemplo la demanda de transporte privado, generando sistemas de monitoreo y evaluación del funcionamiento de redes de trasporte, entre otros; y facilitar los ajustes de la planificación a nuevos escenarios, incorporando métodos de medición de resultados socioeconómicos, entre otros.

El tercer ámbito temático de la PNDU hace referencia al Equilibrio Ambiental, estableciendo como objetivo principal "procurar que el desarrollo de nuestras ciudades... se realice de forma sustentable, equilibrada con el medio na- 
tural, reconociendo y valorando los sistemas en que se insertan" (Ídem: 47).

Entre los propósitos asociados a este objetivo se plantea la conveniencia de considerar los sistemas naturales como soporte en la planificación y diseño de las intervenciones en el territorio, considerando su capacidad de carga, sus particularidades medioambientales y la incorporación de criterios de manejo sustentable del patrimonio natural y sus aspectos paisajísticos, entre otros aspectos; identificar y considerar los riesgos naturales y antrópicos, fomentando las investigaciones y estudios sobre estos, incorporando criterios de reducción de riesgos y estableciendo reglas objetivas para el emplazamientos de construcciones en estas áreas; gestionar eficientemente recursos naturales, energía y residuos, a través de la elaboración de estrategias locales de sustentabilidad y consumo eficiente de energía, incentivando el compromiso y la participación activa de la ciudadanía en la gestión del medioambiente, fomentando el desarrollo e incorporación de tecnologías de eficiencia energética y bioclimática, entre otras; medir y monitorear variables ambientales urbanas, generando catastros integrados de recursos naturales en áreas de intervención, estableciendo metas específicas de mejoramiento ambiental para las ciudades y creando sistemas de indicadores de cumplimiento de objetivos ambientales, entre otros; fomentar el uso sustentable del suelo en ciudades y áreas de expansión, favoreciendo los procesos de reutilización y densificación controlada al interior de las áreas urbanas, reduciendo las externalidades sobre el medio natural y previniendo la ocupación irregular del territorio y los asentamientos informales; fomentar la movilidad urbana a través del uso compartido del espacio público, promoviendo el tránsito peatonal y el uso de la bicicleta, el uso del espacio público para aspectos no funcionales y promoviendo sistemas de transporte público de bajo impacto.

El cuarto ámbito temático está referido a la Identidad y Patrimonio, estableciéndose como objetivo principal "reconocer el patrimonio como bien social, conformado tanto por las obras y manifestaciones de las personas y comunidades como por el entorno natural en que viven" (Ídem: 53).

En tal sentido, se plantea la conveniencia de valorar el entorno físico, construido o natural, formador de la identidad de las comunidades, a través de su incorporación en procesos educativos que permitan valorar la identidad, la cultura y la historia de sus habitantes, propiciando la calidad de la arquitectura como factor de identidad y desarrollo cultural, estableciendo normas y condiciones que promuevan la integración urbanística de proyectos, o desarrollar planes especiales para sectores, ejes viales y espacios públicos que permitan adecuar su diseño y características a las identidades locales, entre otros; valorar la identidad de cada cultura y zona geográfica velando por la coherencia entre las obras y el lugar en que se emplazan, generando sistemas adecuados de participación en la formulación de los Instrumentos de Planificación Territorial, entre otros; conocer, valorar, proteger y gestionar el patrimonio cultural mediante un registro integrado del patrimonio cultural declarado como parte del sistema de información territorial público y actualizado, estableciendo metodologías para su valoración en base a sus atributos y valores, incluyéndolo como factor positivo en la generación de valor social en los sistemas de evaluación social de proyectos, incrementando la asignación de recursos públicos para su salvaguarda, estableciendo mecanismos de compensaciones e incentivos económicos para su protección, entre otros. 
El último de los ámbitos temáticos establecidos en la PNDU cumple un rol articulador de los anteriormente reseñados, resultando fundamental para su operacionalización. Dicho ámbito está referido a la Institucionalidad y Gobernanza, cuyo objetivo central es:

"(...) promover un reordenamiento institucional, tanto de la administración central como de los gobiernos locales, para obtener un sistema integrado y descentralizado de toma de decisiones en materia de desarrollo urbano y territorial que permita la materialización de los postulados de esta Política" (Ídem: 63).

Para su consecución se plantea como central la promoción de un sistema descentralizado de decisiones urbanas y territoriales con fuerte arraigo en el nivel local, lo que implica la provisión de potestades y atribuciones, además de mecanismos de financiamiento solventes, dotación de servicios y equipos técnicos suficientes para su consecución; se propone también la reorganización de potestades públicas en cuatro escalas territoriales: comunal, metropolitana, regional y nacional, cada uno de los cuales deberá elaborar los Instrumentos de Planificación Territorial correspondiente a su ámbito de actuación; asimismo, se propone la elaboración de un sistema de planificación integrado compuesto por planes de ordenamiento territorial, sistemas de financiamiento y decisiones de inversión y herramientas de gestión de carácter multisectorial, descentralizado y participativo; destaca también su consideración de la participación ciudadana efectiva como un derecho fundamental de las personas, para lo cual se propone generar sistemas de participación ciudadana de carácter continuo, organizado, transparente y responsable reconocidos y promovidos institucionalmente; se propone a su vez el desarrollo de un sistema de información territorial único y completo, que contemple un registro consolida- do de la información sobre el territorio y programas de recopilación de información "de abajo hacia arriba"; por último, se propone el desarrollo e implementación de sistemas de medición de la calidad del desarrollo urbano, un sistema expedito de aprobación de iniciativas públicas y privadas, así como también la conformación del Consejo Asesor anteriormente descrito encargado de propiciar la continuidad, permanencia e implementación de esta Política.

Tomar como punto de referencia el caso de la PNDU permite visualizar un cierto estado de la discusión y el debate sobre desarrollo urbano en nuestro país y conocer los puntos de vista de los expertos en la materia. Sin embargo, ello también permite abrir miradas críticas sobre los temas y enfoques emergentes señalados en ella.

En tal sentido, y si bien muchos de los objetivos y lineamientos presentes en cada uno de estos ámbitos resultan tremendamente necesarios y relevantes para el desarrollo de los centros urbanos en nuestro país, es necesario establecer algunas consideraciones críticas respecto de ellos. Por una parte, su formulación da cuenta someramente de las condiciones políticas, económicas y socioculturales del contexto bajo las cuales se busca implementar esta Política, sin denotar mayor análisis crítico de los enfoques, expectativas e intereses de quienes han participado en su formulación y actual proceso de implementación. Asimismo, predomina en ella una perspectiva "de arriba hacia abajo", con énfasis en el conocimiento y la perspectiva de los expertos de marcada racionalidad urbanística desde la cual se han formulado objetivos y lineamientos, donde escasamente se reconocen las experiencias y prácticas sociales de los habitantes, en su gran mayoría alejadas de la ya mencionada racionalidad urbanística. Por último, dichos objetivos y lineamientos parecen más enfocados 
a la intervención de un objeto que a la consecución de un proceso, contemplando escasamente la reflexividad como recurso cognoscitivo y político relevante para dar cuenta de las complejidades, incertidumbres y tensiones que aparecerán en su proceso de implementación. Justamente es en torno a estas consideraciones que la urbanidad como campo de conocimiento puede constituir un interesante aporte y complemento a la manera como se formulan e implementan los alcances de la PNDU.

\section{Algunas consideraciones críticas en torno a la PNDU}

De acuerdo a lo planteado por Prat (2013), el proceso de formulación e implementación de la PNDU pone en evidencia la relevancia de la política en la conformación de los centros urbanos en nuestro país, constatación invisibilizada durante las últimas décadas a través de una "no-política de hechos consumados" que ha predominado en nuestros procesos de desarrollo urbano facilitando el predominio de los agentes de mercado. No obstante ello, el autor reseña que ésta no ha sido clara en empoderar suficientemente al Estado y a la sociedad organizada como agentes sociales.

Sin embargo, y tal como se planteaba anteriormente, una primera consideración crítica a la formulación y actual impementación de la PNDU es su vaguedad respecto de los enfoques ideológicos que sustentan sus objetivos y lineamientos, componentes fundamentales si se trata de discutir sus marcos de referencia. En tal sentido, la PNDU aparece enmarcada en el híbrido cruce entre los principios de equidad, competitividad y sostenibilidad provenientes de distintos modelos de desarrollo urbano.
El principio de equidad deriva de los resabios del modelo de desarrollo keynesiano-fordista predominante en las sociedades occidentales desde la crisis económica del año 1929 y cuyo profuso cuestionamiento se inicia a mediados de la década de 1970. En el caso chileno, aunque el sector público concentró la mayor parte de las competencias urbanísticas durante dicha época, los principios de equidad y cohesión social propiciados por este modelo no lograron implementarse completamente. $Y$ aunque en la actualidad este modelo ha perdido muchas de sus atribuciones reguladoras sobre el crecimiento económico y el bienestar social, sigue siendo reconocido como el principal referente en la promoción de políticas de desarrollo y en la articulación de una mayor coordinación entre el Estado, los sectores privados y la sociedad civil.

Por su parte, la competitividad se ha constituido en el principio actualmente predominante gracias a la consolidación institucional del modelo neoliberal-posfordista desde mediados de los años setenta hasta la actualidad. Esta tendencia a la competitividad ha avanzado de la mano con la implementación de un nuevo paradigma tecnológico, de un nuevo régimen de acumulación y de un nuevo modo de regulación orientados hacia el constante aumento de la productividad. Aunque este modelo también ha sido incompletamente adoptado en nuestro país - no obstante su experiencia como laboratorio de dicho modelo - algunas de sus características sí han sido implementadas: disminución en las atribuciones reguladoras del sector público, una progresiva desregulación urbanística y un importante ascenso del sector privado como agente urbano, entre otros.

Por último, las tendencias hacia la sustentabilidad han avanzado a partir de la emergencia de un modelo alternativo a los dos anteriores, 
el cual se sustenta no solamente en posiciones críticas respecto de estos sino también en propuestas novedosas que apuntan a la movilización de recursos endógenos articulados en sistemas territoriales de producción. En aras de este objetivo, estos modelos propician la promoción del aprendizaje localizado, la construcción de redes de asociación y la conformación de una base institucional regional, las cuales involucren e integren a toda la comunidad regional. Aunque en nuestro país este modelo se encuentra actualmente en ciernes, ha ganado importante influencia principalmente entre organizaciones de la sociedad civil.

Comprender la urbanidad implica observar cómo estos tres principios se plasman en la vida cotidiana de los habitantes urbanos, y por tanto, cómo ellos se reproducen en su vida social. Asimismo, ella permite visibilizar los enfoques, intereses y expectativas de los agentes sociales urbanos ponen en juego al momento de participar en su proceso de formulación e implementación. En tal sentido, una atención más acabada a la urbanidad como campo de relaciones de dichos agentes y sus intereses constituye una oportunidad insoslayable para vislumbrar las posibilidades de consenso y apropiación de las orientaciones emanadas de la Política.

La segunda consideración crítica respecto de su formulación y actual implementación es el predominio de una perspectiva "de arriba hacia abajo", con énfasis en el conocimiento y la perspectiva de los expertos de marcada racionalidad urbanística desde la cual se han formulado objetivos y lineamientos, donde escasamente se reconocen e incorporan las experiencias y prácticas de los habitantes urbanos, principalmente marcadas por la espontaneidad e informalidad en la producción del hábitat y el territorio. En tal sentido, no cabe duda que la promoción de una perspectiva "de abajo hacia arriba" para el desarrollo urbano necesariamente debe considerar la urbanidad como recurso constitutivo.

Esta consideración se encuentra en consonancia con el planteamiento desarrollado por Dockendorff (2013), para quien la ciudad constituye un "espacio político" donde coexisten fuerzas, intereses y transacciones entre distintos agentes sociales urbanos, los cuales pueden entrar en relaciones de conflicto, cooperación o coordinación. Hasta entrada la década de 1990 el origen político de las decisiones urbanas radicaba en una pasiva connivencia entre los agentes sociales representantes del Estado y del mercado, situación que durante los últimos años ha cambiado fuertemente debido a la emergencia de un nuevo actor en el arreglo de poder de la sociedad como son los ciudadanos, quienes cada vez con mayor fuerza buscan promover sus derechos civiles y territoriales. En tal sentido, el gran desafío de la PNDU radica, a juicio de este autor, en integrar a los ciudadanos a la formulación de un proyecto de ciudad cuyo sentido democrático y sustentable sea la base de su estabilidad y proyección futura.

La tercera consideración crítica respecto de la formulación y actual implementación de la PNDU es su escasa referencia a la reflexividad para analizar críticamente la noción de "desarrollo urbano" y sus particulares connotaciones políticas, económicas, sociales y culturales. Tal como plantea Elizalde (2005), el concepto de desarrollo surge vinculado al esfuerzo político por hacer transitar a los países pobres por un camino que les permita superar su pobreza o carencia de desarrollo. En tal sentido, no solo da cuenta de un estado sino también de un proceso, los cuales adquieren significación y relevancia bajo la lógica del progreso lineal promovido en el transcurso de la modernidad. Por 
su parte, la noción de "desarrollo urbano" hace alusión tanto a los procesos de urbanización como a las transformaciones socioeconómicas que éstos generan sobre la población (Grupo ADUAR, 2000). En ambos sentidos, esta noción sugiere un mejoramiento en la calidad de vida de la población, situación que no necesariamente se cumple en la totalidad de los casos.

Sin embargo, en el caso chileno los procesos de desarrollo urbano poseen una constitución paradójica que se desprende de la misma construcción de nuestra modernidad y que se manifiestan en una compleja e inacabada transición desde ciudades a urbes, y donde la articulación propuesta por Capel (Op.cit.) entre polis, urbs y civitas nunca llega a plasmarse suficientemente. Las unidades político-administrativas representadas en la polis se constituyen principalmente como reductos de las elites, promoviendo principios y modelos de desarrollo que no necesariamente logran ser entendidos, apropiados y debatidos por el resto de la población, restringiendo de esta manera su debate democrático. Por su parte, el espacio construido representado en la urbs se vuelve expresión y recurso de dichos modelos de desarrollo, observándose una implementación segregada y desigual de los mismos, promoviendo procesos de exclusión social respecto de su apropiación y participación ciudadana. En lo que respecta a la vida social representada en la civitas, su diversidad y heterogeneidad, muchas veces mediada por el conflicto, termina desbordando los ámbitos anteriores y con ello frustrando la constitución de un proyecto de desarrollo colectivo e integrador. Este paradójico panorama presenta una marcada continuidad desde el modelo de ciudad pre-industrial hasta el actual estado de "ciudades en proceso de globalización" (De Mattos, 2000).
Considerando que las sociedades latinoamericanas nunca lograron conformar y consolidar completamente el modelo fordista desarrollado en las principales sociedades capitalistas, desde fines de la década de 1970 los países de la región se han visto impulsados progresivamente a adaptarse al modelo de desarrollo posfordista en formación, detentando los mayores niveles mundiales de desigualdad social y concentrando en su interior importantes y crecientes focos de pobreza y exclusión social. Estas siguen su devenir histórico más como agregados de formas, funciones y significados que como urbes, generando una constante tensión entre las políticas y estrategias de urbanización implementadas principalmente por el Estado y agentes privados de corte empresarial, y aquellas prácticas sociales de carácter cotidiano, espontáneo, no planificadas desarrolladas por sus habitantes (Ducci, 2000). En tal sentido, atender a la relevancia que la urbanidad posee en la actualidad implica avanzar en la promoción de una perspectiva más comprensiva, crítica y reflexiva sobre nuestros paradójicos procesos de desarrollo urbano, así como también implica una mirada más atenta a las relaciones entre los distintos agentes sociales urbanos y sus prácticas sociales características.

\section{Relevancia de la urbanidad para abordar procesos de desarrollo urbano}

Comprender la urbanidad ha sido un objetivo de las ciencias sociales que posee antigua data, arraigándose principalmente en enfoques culturalistas como los desarrollados por G. Simmel, F. Tönnies y la Escuela de Chicago. Sin embargo, y no obstante su permanente actualidad, llama particularmente la atención la revalorización que la urbanidad está teniendo actualmente de la mano de otros dos referentes históricos como son Henri Lefebvre y Jane Jacobs. 
En el caso de Lefevbre, este constituye un referente fundamental en la formulación de los enfoques críticos a los modelos de urbanización neoliberal, alcanzando en la actualidad una importante influencia sobre distintas iniciativas de alcance global como son por ejemplo la Carta Mundial por el Derecho a la Ciudad y la Vivienda, el Foro Social Mundial, y diversos movimientos sociales críticos al modelo de desarrollo urbano neoliberal. En tal sentido, uno de los principales aportes que este enfoque ha realizado al debate en torno a las políticas de desarrollo urbano es su preocupación por investigar las condiciones sociales, políticas, económicas y culturales presentes en estos procesos y a la vez revalorizar la importancia de los agentes sociales y de sus prácticas urbanas cotidianas como promotores y generadores de urbanidad. Destaca en este sentido su preocupación por las prácticas urbanas cotidianas, entendidas como el conjunto de comportamientos y conductas que caracterizan las distintas formas de civismo, sociabilidad y urbanidad actualmente existentes.

En el caso de Jacobs, su influencia se hace patente en la formulación de enfoques emergentes, los cuales recogen influencias de planteamientos evolucionistas, organicistas y de las teorías de la complejidad. Uno de los aspectos más relevantes de este enfoque es su carácter transdisciplinario, dentro del cual se conjugan aportes provenientes desde las ciencias naturales, las ciencias informáticas y también las ciencias sociales, lo cual ha captado rápidamente el interés de las nuevas generaciones de profesionales. Destaca también la relevancia otorgada a la interacción social en la vida cotidiana y su expresión en programas de simulación y modelado, que visibilizan la importancia de las redes sociales para el intercambio constante y continuo de nuevas experiencias entre distintos agentes sociales tanto en sus entornos locales y como en contextos globales, así como también su importancia para el desarrollo de iniciativas con importantes componentes ciudadanos como por ejemplo el urbanismo táctico.

Si bien los enfoques críticos y emergentes presentan una serie de divergencias de carácter epistemológico, teórico y metodológico, es posible encontrar en ellos un importante punto de convergencia: su reconocimiento fundamental a la relevancia que las prácticas sociales urbanas tienen como productoras de urbanidad y su importancia para la creación, transformación y apropiación del espacio urbano. Dicha relevancia se sustenta en varias consideraciones. En primer lugar, ellas son expresión de los agentes y actores sociales, quienes las crean y recrean en un tiempo-espacio social determinado y por tanto tienen un carácter situado en referencia a su contexto histórico-social; asimismo, guardan una estrecha relación con la revalorización de la vida cotidiana, constituyendo un ámbito de articulación mesosocial entre perspectivas microsociales y perspectivas macrosociales; destaca también su carácter eminentemente relacional y por tanto dinámico y transformador de las condiciones de vida de los habitantes urbanos, por lo que no es posible presuponerlas estáticas ni inamovibles; poseen además un carácter irreductible a procesos de institucionalización, conteniendo el germen de la contradicción y del desbordamiento a la norma adquiriendo un potencial creativo irrenunciable. En síntesis, atender a las prácticas sociales urbanas permite acceder al potencial creativo e innovador de la urbanidad, avanzar más allá de la institucionalidad de los procesos de desarrollo urbano y vislumbrar sus dimensiones instituyentes.

Otro ámbito de reconocimiento a la importancia que la urbanidad y sus prácticas sociales 
tienen para el desarrollo urbano puede vislumbrarse en un progresivo cambio de enfoque en las políticas urbanas actualmente impulsadas por la Unión Europea. De acuerdo con Gatti (2007), estas presentan una creciente valorización de la interacción social en ámbitos locales de actuación urbanística. En este sentido, la autora destaca la relevancia otorgada a la compresenza en la medida que ella permite sustentar las políticas urbanas desde las prácticas sociales de urbanidad lo que a su juicio "implica la consideración de la sociedad como un sujeto capaz de autorregularse, un sujeto capaz de planificar, de hacer política y políticas aunque sea de manera no intencional" (Gatti, 2007: 6).

En este sentido, para Gatti la revalorización de la urbanidad y sus prácticas sociales conllevan un cuestionamiento y replanteamiento de la manera como tradicionalmente se concibe la política desde el urbanismo, principalmente porque cuestiona el carácter a priori, estático y fijo del territorio, pasando este a constituirse en términos relacionales en la medida que las prácticas sociales no necesariamente aparecen delimitadas y restringidas a límites estáticos ${ }^{4}$.

La constatación planteada por Gatti es refrendada por el Informe de la Comisión de las Comunidades Europeas denominado Ciudades del mañana: retos, visiones y caminos a seguir (2011), el cual promueve un enfoque de gobernanza multiescalar donde se asigna particular reconocimiento a la importancia que la comunidad local tiene como eje del desarrollo urbano, aplicándose principalmente a nivel barrial. Sobre esta base, el Informe propone un mayor impulso al establecimiento de partenariados entre entidades públicas, privadas y "voluntarias" a nivel local, las cuales puedan movilizar e implicar a las comunidades y organizaciones locales en proyectos de desarrollo urbano. En tal sentido, el establecimiento de partenariados locales es promovido como herramientas de desarrollo complementarias a aquellas tradicionalmente utilizadas por los gobiernos locales para alcanzar el compromiso de los distintos agentes sociales involucrados. Para tales efectos, el Informe asigna particular importancia al desarrollo de capacidades a nivel local que permitan nivelar y equilibrar los recursos que los distintos agentes sociales poseen para participar en ellos, incorporando la diversidad de grupos socioeconómicos, étnicos, etarios, de género, existentes. De esta manera el Informe busca prever la excesiva influencia que aquellos agentes con un mayor acceso a la información y/o capacidad de representación puedan alcanzar en procesos de desarrollo urbano.

Otra importante expresión de la importancia que la urbanidad y sus prácticas sociales tienen en la actualidad lo constituye la consolidación de nuevas formas de organización y participación social. Ejemplos de esto lo constituye la conformación de grupos sociales con identidades flexibles, sentimientos de pertenencia aleatorios y formas de organización informales, como son las "redes sociales espacialmente dispersas" propuestas por Cucó Giner (2004) o las "movilizaciones" propuestas por Delgado (2007). Estas últimas destacan por su capacidad colectiva de apropiación de espacios urbanos, donde la multitud se vuelve "fusional" expresándose a través de fiestas y celebraciones religiosas, conmemoraciones ciudadanas, manifestaciones políticas, eventos deportivos, etc. ${ }^{5}$

Las consideraciones anteriormente reseñadas constituyen expresión del paulatino reconocimiento a la relevancia que la urbanidad y sus prácticas sociales ha alcanzado en los actuales 
procesos de desarrollo urbano. En este sentido, el progresivo protagonismo que estas formas de organización social abiertas y flexibles han ido adquiriendo en los procesos de desarrollo urbano han sido abordadas por el mismo Delgado a través de la relación entre cultura urbana y cultura urbanística, que a nuestro entender resulta fundamental para avanzar en una mayor complementariedad entre ciencias sociales y urbanismo. Tomando como referencia la distinción propuesta por Lefebvre entre el "espacio percibido", el "espacio pensado" y el "espacio vivido", Delgado plantea que mientras la cultura urbana refiere a "la ciudad practicada" por parte de sus habitantes, la cultura urbanística referencia a "la ciudad concebida" por urbanistas-planificadores, poniendo en evidencia una distinción aún más profunda que las anteriores y que dice relación con orden y control v/s desorden y espontaneidad. Este desorden y espontaneidad, que en muchas ocasiones y con distinta magnitud desborda el ordenamiento y control urbanístico está radicado a juicio del autor en los practicantes de la ciudad, es decir, sus habitantes ${ }^{6}$.

Siguiendo a Delgado, el paso desde una distinción a una aparente tensión y/o confrontación permanente entre orden-control / desorden-azar estaría radicada en una mala concepción de la práctica urbanística, la cual tendría por objetivo dominar y organizar lo urbano. Sin embargo, para el autor dicha pretensión resulta equívoca en la medida que lo urbano está en permanente conformación, nutriéndose de diferencias y diversidades, acuerdos y conflictos, y por lo tanto abierta a su negociación, innovación, sorpresa y tensión permanentes.

Esta interrogante resulta relevante atendiendo a lo que distintos autores han identificado como el surgimiento y conformación de una nueva cultura urbanística, con un marcado carácter ciudadano y revalorización de formas de auto organización y autogestión sustentadas fuertemente en prácticas sociales de urbanidad. Esta nueva cultura urbanística destaca por la incorporación y consideración de aquellos habitantes urbanos - individuales y colectivos - que aparecen ocupando posiciones alternativas dentro del entramado político tradicional, pero que poseen importantes capacidades para incidir e intervenir en la organización del espacio. Muchos de ellos ni siquiera responden a las formas de organizaciones tradicionales representadas en ONG, cooperativas o movimientos sociales asociativos, por lo que entender su importancia implica desarrollar formas de observar y de dar visibilidad a aquellas actuaciones que no necesariamente son estratégicas ni emblemáticas, sino que se desarrollan bajo la dinámica de las prácticas sociales urbanas.

En el campo del urbanismo, distintos autores han tomado nota de la paulatina conformación de una nueva cultura urbanística, revalorizando las capacidades de autogestión y auto organización que históricamente los habitantes han exhibido en los centros urbanos. Vergara \& De las Rivas (2004), por ejemplo, han destacado la importancia que las smart communities han tenido en procesos locales de participación social urbana y particularmente del compromiso colaborativo que éstas manifiestan, alejándose del prototipo del habitante urbano caracterizado por su individualismo, su nomadismo y su desarraigo. De acuerdo con los autores, las claves para la formación de "comunidades creativas" no se encuentran en grandes postulados abstractos ni teóricos, no es necesario inventarlos ni crearlos ex profeso, sino más bien pueden rastrearse y encontrarse en la vida cotidiana de las personas. Esta idea, desarrollada por distintos pensadores críticos del urba- 
nismo racionalista tanto desde la arquitectura, el urbanismo como desde las ciencias sociales, resulta de gran interés en la medida que rescata la importancia de los espacios de vida cotidiana y rastrea las capacidades presentes en la vida habitual de la calle, en el barrio, "en la que existen muchas estrategias sociales eficientes de autorregulación, fundadas en la colaboración y no en la exclusión" (Vergara \& De las Rivas, 2004:143). Este planteamiento se acerca al concepto de "tercer espacio" propuesto por Soja a instancias de Lefebvre. Vergara \& De las Rivas lo consideran como una perspectiva importante y necesaria para la construcción del espacio urbano metropolitano, idea que se plasma en la articulación entre participación e innovación?.

Por su parte, Innerarity (2006) asume esta tendencia como parte de una "tercera vía" que desde fines de la década de 1990 ha surgido como alternativa a los modelos políticos que transitan entre el "estatismo" heredado del modelo keynesiano y aquel enfoque empresarial de corte neoliberal. Si bien esta "tercera vía", a juicio del autor, reivindica "valores" sociales como la cooperación, la integración, la confianza y la legitimación entre los actores y agentes sociales, no significa una renuncia completa del papel que el Estado juega en la organización de la sociedad ni tampoco conlleva una instrumentalización privada del ámbito estatal como sugieren ciertas concepciones del management.

Por su parte, Freire (2009) articula estas tendencias en torno al concepto de urbanismo ciudadano o emergente. Esta propuesta enmarcada en el avance de las sociedades del conocimiento está orientada a reconocer y fortalecer la importancia que los ciudadanos organizados en redes sociales pueden tener en el desarrollo de un urbanismo más participativo, el cual se nutra de las tecnologías digitales actualmente disponibles para participar activamente en la gestión de los sistemas de información y toma de decisiones. En tal sentido, y cuestionando la hermética influencia que muchas veces alcanzan los grupos de expertos en estas materias, el autor propone el desarrollo de un modelo emergente o P2P que favorezca la conformación de redes distribuidas de intercambio y colaboración urbana ${ }^{8}$.

Tres elementos resultan interesantes a destacar de la propuesta de Freire: por una parte, la necesidad de promover una alternativa al urbanismo convencional o tradicional, sustentada en la emergencia de la ciudadanía; por otra, la importancia asignada a las redes sociales digitales como medio para fomentar la capacidad de acción y participación en la toma de decisiones; por último, la necesidad, y a la vez la posibilidad, de sustentar proceso colaborativos basados en los supuestos de emergencia. Asimismo, y aludiendo a los aportes realizados por Appadurai, el autor llama la atención en torno a tres condiciones que a su juicio propician la emergencia de una participación ciudadana activa: la información en sus diversos usos y formatos, los "espacios de comunicación" tanto analógicos como digitales, y la capacidad de toma de decisiones con que cuentan los ciudadanos en la actualidad.

En síntesis, y tal como puede desprenderse de los autores anteriormente reseñados, comprender la urbanidad puede constituir un importante aporte y aliciente para la conformación de una nueva cultura urbanística también en nuestro país que incida en la implementación de nuestra PNDU.

\section{Conclusiones. Oportunidades y desafío para la antropología urbana}


Tal como lo expresa en su introducción, el presente artículo plantea la idea de que no obstante su escasa relevancia en los procesos de desarrollo urbano en general y en la formulación e implementación de la PNDU en particular, es posible encontrar un interesante ámbito de oportunidades y desafío para el desarrollo de la antropología urbana en Chile.

Dichas oportunidades y desafíos se articulan en torno al reconocimiento que la urbanidad tiene en la conformación del campo de conocimiento urbano. Si bien desde las ciencias sociales, y en particular desde la antropología urbana, esta ha sido considerada un componente fundamental de nuestras ciudades en la medida que involucra directamente las experiencias y prácticas sociales de los habitantes urbanos, ella ha tenido escasa consideración desde el urbanismo, situación que en la actualidad está cambiando paulatinamente en la medida que existe un progresivo reconocimiento de su importancia para incorporar activa y participativamente a los habitantes urbanos en dichos procesos.

En este sentido, un primer ámbito de oportunidad alude a la exploración de un objeto de estudio que desborda los límites disciplinares y que permite a la antropología urbana participar de manera activa en la conformación de un campo de conocimiento en torno a lo urbano donde convergen otras disciplinas de las ciencias sociales como la sociología y la geografía, y que se amplía a otros ámbitos del conocimiento como la arquitectura, la economía, el derecho, el urbanismo, entre otras.

Un segundo ámbito de oportunidad refiere a la ampliación de los campos de conocimiento y actuación de la antropología urbana hacia otros más aplicados como la planificación y gestión urbana, de los cuales ha estado tradicionalmente distanciada. Esto permitiría ampliar progresivamente su incidencia tanto en procesos de desarrollo urbano como en la formulación de políticas públicas, las cuales muchas veces requieren enfoques e instrumentos teóricos y metodológicos que les permitan avanzar hacia un mayor conocimiento y vinculación con los habitantes urbanos.

Lo anterior se relaciona directamente con un tercer ámbito de oportunidades como es la creciente demanda por enfoques teóricos y recursos metodológicos que permitan orientar y desarrollar procesos participativos "de abajo hacia arriba", las cuales permitan articular consensos y compromisos entre los distintos agentes sociales urbanos e incorporar activamente a la amplia diversidad de comunidades y organizaciones ciudadanas progresivamente avanzan en la defensa de sus derechos civiles y territoriales.

Por último, y como corolario de los anteriores, un cuarto ámbito de oportunidad lo constituye el potencial aporte de la antropología urbana a la paulatina y emergente conformación de una nueva cultura urbanística sustentada justamente en una mayor interacción entre la característica racionalidad técnica de los urbanistas más tradicionales con una creciente preocupación por las prácticas sociales de los habitantes urbanos, abriéndose a la participación desde perspectivas más abiertas y flexibles que las actualmente predominantes.

Este conjunto de ámbitos de oportunidad, así como otros posibles, requieren de un desafío consustancial: la necesidad de avanzar hacia la conformación de enfoques teóricos y metodológicos más críticos, comprensivos y principalmente más reflexivos sobre los procesos de desarrollo urbano. Críticos, en la medida 
que permitan visualizar con claridad la importancia de los contextos donde se implementan los procesos de desarrollo urbano y analizar en profundidad las condiciones de producción de conocimiento e intervención existentes en éstos. Comprensivos, en la medida que permitan reconocer en dichos contextos las prácticas sociales y las experiencias de los habitantes urbanos, complementando la primacía que en la actualidad poseen los enfoques expertos respecto de las formas como deben orientarse e implementarse los procesos de desarrollo urbano. Reflexivos, en la medida que permitan identificar y abordar las complejidades y paradojas que han marcado y siguen marcando el desarrollo urbano en nuestras ciudades, muchas de las cuales son expresamente invisibilizadas desde las esferas institucionales. En síntesis, enfoques más críticos, comprensivos y reflexivos constituyen recursos fundamentales para promover la observación, la visibilidad y el reconocimiento de aquellas dimensiones socioculturales fundamentales para el desarrollo de las ciudades.
Lo anterior exige avanzar desde la descripción del caso exótico que tradicionalmente ha imperado en nuestra disciplina hacia el análisis de los procesos, perspectiva que muchas veces nos resulta un tanto ajena. Esto alude no solamente a la producción de conocimiento, sino que también, y preponderantemente, alude a los ámbitos de decisión política sobre los centros urbanos. Tal como plantean Montaner y Muxí:

“(...) la primera decisión política - en cualquier actividad de teoría, historia y crítica del arte y la arquitectura - radica en lo que se visibiliza y en lo que se ignora, en lo que se promueve y en lo que se oculta, en lo que se dice y en lo que se calla y a quién se silencia" (Montaner \& Muxí, 2011: 16).

En este sentido, la voluntad política por identificar, visibilizar y reconocer las nuevas ideas y formas de hacer las cosas en las sociedades urbanas contemporáneas, constituye una interrogante siempre abierta a la que no debemos dejar de atender.- 


\section{Notas}

${ }^{1}$ Nuestra concepción de la urbanidad se acerca a aquella desarrollada por la autora, destacando su “(...) capacidad de combinar el reconocimiento del otro con la reserva y el distanciamiento, la capacidad de tratar lo desconocido como si fuera uno y lo conocido como si fuera otro. Es una mezcla sui generis de lejanía y proximidad, de interés y de indiferencia, que hace posible la convivencia pacífica entre seres distintos" (Giglia, 2001: 803).

${ }^{2}$ Tal como establece el autor, "(...) la urbanidad designa más el trabajo de la sociedad urbana sobre sí misma que el resultado de una legislación o de una administración, como si la irrupción de lo urbano en el discurso sociológico estuviera marcada por una resistencia a lo político" (Joseph, 2002:28).

3 "El objetivo principal de esta Política es generar condiciones para una mejor "Calidad de Vida de las Personas", entendida no solo respecto de la disponibilidad de bienes o condiciones objetivas sino también en términos subjetivos, asociados a la dimensión humana y relaciones entre las personas.

Esta Política se basa en el concepto de "Desarrollo Sustentable", entendiendo desarrollo como el aumento de las posibilidades de las personas y comunidades para llevar adelante sus proyectos de vida en distintos ámbitos. Y sustentable, en términos "que la satisfacción de las necesidades actuales de las personas se realice sin comprometer la capacidad de las futuras generaciones para satisfacer las suyas" (MINVU, 2014:16). En cuanto al territorio, esta Política considera que este no es un bien cualquiera sino que tiene la cualidad de único e irrepetible, por lo que no resulta apropiado referirse a él exclusivamente en términos de escasez o abundancia. Su utilización, cuando se trata de fijar usos permanentes, debe ser objeto de regulación, bajo el principio del "Bien Común" y el respeto de los derechos individuales" (Ibídem).
${ }^{4}$ Gatti establece que en el marco de esta reformulación cobran sentido la distinción realizada por Lefebvre entre el "espacio percibido" - aquel sustentado en las prácticas sociales rutinarias y creativas-, el "espacio pensado" por parte de los planificadores - representaciones del espacio -, y el "espacio vivido" por parte de los habitantes, en contextos de dominación y alienación, así como también la importancia asignada por M. de Certeau a las prácticas sociales que subvierten la lógica urbanística racionalista y abstracta, lo cual se expresa en su distinción entre "estrategias" y "tácticas".

${ }^{5}$ De acuerdo con el autor, las movilizaciones urbanas pueden ser entendidas como "dispositivos de reificación de identidades habitualmente negadas en la vía pública", lo cual supone un reconocimiento de diferencias en un contexto de igualación e invisibilización cultural. (Ídem: 165).

6 "Por doquier, constantemente, podemos dar con pruebas de la actualidad de un viejo contencioso inherente a la historia misma de la ciudad moderna: la que opone el conjunto de maneras de vivir en espacios urbanizados - la cultura urbana propiamente dicha - a la estructuración de las territorialidades urbanas, es decir la cultura urbanística" (Delgado, 2007: 11).

7 "El tema de las comunidades creativas es clave para las Smart Communities, entendidas como un conjunto de experiencias destinadas a fomentar el desarrollo económico, el crecimiento del empleo y un incremento de la calidad de vida locales. Son consecuencias de la iniciativa pública o de asociaciones locales que parten del convencimiento de que para lograr sus fines es necesario un impulso colectivo" (Vergara \& De las Rivas, 2004: 141).

8 "El urbanismo emergente se contrapone, o al menos complementa, a la planificación urbanística convencional. Lo emergente surge en gran medida de modo auto-organizado como consecuencia de la interacción y colaboración de grupos humanos amplios y diversos, como los que habitan las ciudades. En este sentido, la participación ciudadana surge como motor del proceso, pero entendida no solo como debate y deliberación, sino especialmente como acción directa en la "construcción" de la ciudad" (Freire, 2009: 19). 


\section{Referencias bibliográficas}

Capel, H. (2003). "A Modo de Introducción: Los problemas de las ciudades. Urbs, Civitas y Polis". En Capel, H. (coord.). Ciudad, Arquitectura y Espacio Urbano. Almeria: Instituto de Estudios Cajamar, pp. 9-24.

Comisión de las Comunidades Europeas. Dirección General de Política Regional (2011). Ciudades del mañana: retos, visiones y caminos a seguir. Luxemburgo: Oficina de Publicaciones Oficiales de las Comunidades Europeas.

Cucó Giner, J. (2004). Antropología urbana. Barcelona: Ariel.

De Mattos, C. (2000). "Santiago de Chile, globalización y expansión metropolitana: lo que existía sigue existiendo". Seminario "Efectos de la globalización en la evolución del Santiago Metropolitano". Santiago: Pontificia Universidad Católica de Chile.

Delgado, M. (2007). Sociedades Movedizas. Barcelona: Anagrama.

Dockendorff, E. (2013). "La política habita la ciudad: La descentralización del Estado como requisito de la experiencia democrática de ciudad”. En López, E., Arriagada, C., Jirón, P. \& Eliash, H. (eds). Chile Urbano hacia el Siglo XXI. Santiago: Editorial Universitaria, pp. 35-40.

Ducci, M. E. (2000). "Santiago: territorios, anhelos y temores. Efectos sociales y espaciales de la expansión urbana". EURE, vol.26, № 79 , pp.5-24.

Elizalde, A. (2005). "Desarrollo". En Salas, R (ed.) Pensamiento Crítico Latinoamericano. Conceptos Fundamentales. Santiago: Universidad Católica Silva Henríquez, pp. 467-496.

Freire, J. (2009). "Urbanismo emergente: ciudad, tecnología e innovación social - Emerging urban planning: city, technology and social innovation". En Paisajes Domésticos / Domestic Landscapes, Madrid: Ed. SEPES, pp. 18-27.
Gatti, C. (2007). "El rol del concepto de 'prácticas sociales' en el análisis de la producción del espacio común". En Cuartas Jornadas de Jóvenes Investigadores. Buenos Aires: Instituto de Investigaciones Gino Germani.

Giglia, A. (2001). "Sociabilidad y Megaciudades". Estudios Sociológicos, XIX: 57, pp. 799-821.

Grupo Aduar (2000). Diccionario de geografía urbana, urbanismo y ordenación del territorio. Barcelona: Ariel.

Imilan, W. \& Lange, C. (2003). "Aproximación a la antropología urbana chilena: lo urbano y el trabajo de campo en la ciudad". En Richard, N. (ed). Movimiento de campo: en torno a cuatro fronteras de la antropología en Chile, Guatemala: ICAPI, pp. 27 - 44.

Innerarity, D. (2006). El Poder Cooperativo: Otra Forma de Gobernar. Barcelona: ESADE.

Joseph, I. (1988). El transeúnte y el espacio urbano: ensayo sobre la dispersión del espacio público, Buenos Aires: Gedisa.

Ministerio de Vivienda y Urbanismo (2014). Política Nacional de Desarrollo Urbano. Ciudades Sustentables y Calidad de Vida. Santiago: Ministerio de Vivienda y Urbanismo.

Montaner, J. M. \& Muxí, Z. (2011). Arquitectura y Política. Ensayos para mundos alternativos. Barcelona: Ed. Gustavo Gili.

Prat, L. (2013). "La propuesta de la Universidad de Chile a la Política Urbana desde la investigación sobre la ciudad y los barrios, en perspectiva del interés público y sus habitantes". En López, E., Arriagada, C., Jirón, P. \& Eliash, H. (eds). Chile Urbano hacia el Siglo $X X I$. Santiago: Editorial Universitaria, pp. 11-18.

Vergara, A. \& De las Rivas, J. L. (2004) Territorios Inteligentes. Nuevos Horizontes en el Urbanismo. Madrid: Fundación Metrópoli. 